\title{
THE DETERMINATION OF THE CARDIAC OUTPUT IN MAN AT BRIEF INTERVALS BY A MODIFICATION OF THE ETHYL IODIDE METHOD ${ }^{1}$
}

\author{
By J. C. SNYDER 2 \\ (From the Surgical Laboratories of the Harvard Medical School at the Massachusetts \\ General Hospital, Boston)
}

(Received for publication April 1, 1938)

Ethyl iodide has been used extensively for the determination of cardiac output in man by the method of Starr and Gamble $(1,2,3)$. In their procedure, the subject breathes from a spirometer for a total of 25 or 30 minutes during a determination in duplicate. Under many conditions a more rapid technique is required. For example, surgical patients may frequently be too sick in the first few days after operation to undergo repeated determinations involving a long period of breathing through mouthpiece and valves. Moreover, the rapidity of the circulatory changes during recovery from anesthesia demands a method of study which occupies less time than 15 to $20 \mathrm{~min}$ utes for a single estimation. This is particularly true of the changes induced by emotion or vigorous exercise.

Analysis of the air samples for a determination in duplicate by Cool's iodate technique (4) takes approximately 2 hours. The method is thereby restricted to very few determinations in any one day.

This report includes three sections. Section I describes a modification of Starr and Gamble's method which permits a determination in duplicate of cardiac output in 12 minutes, and additional determinations every 6 minutes thereafter. Section II presents a method of sampling which requires 45 minutes for the analyses involved in duplicate determinations, or 1 hour for triplicate determinations. Section III contains the data obtained on normal subjects and hospital patients using the modifications as described in Sections I and II.

\section{MODIFICATION OF STARR AND GAMBLE'S METHOD}

\section{A. Alveolar air}

(The reader is referred to Starr and Gamble's papers for a description of the principles and technical details of their method.)

1 Aided by grants from the Josiah Macy, Jr. Foundation and the William F. Milton Fund.

2 Research Fellow in Surgery.
In Starr and Gamble's procedure, the negative pressure during inspiration is utilized to withdraw the last few cubic centimeters of each expiration into a $500 \mathrm{cc}$. tube. A Bohr meter in the alveolar circuit is used to regulate the rate of collection of the sample. Approximately $21 / 2$ liters of air must be drawn through before constant composition is attained in the $500 \mathrm{cc}$. tube, the time required being about 12 to 15 minutes.

The principal change is a reduction of deadspace in the alveolar circuit from $500 \mathrm{cc}$. to $15 \mathrm{cc}$. and a consequent reduction in the washing-out time from 15 minutes to 20 seconds. This is accomplished with the apparatus illustrated in Figure 1. A measured adjustable volume of mercury is allowed to flow out of a sampling tube during each inspiration, withdrawing the last portion of the previous expiration from the mouthpiece (1, Figure 4). Mercury is used instead of water to prevent loss of ethyl iodide and to facilitate rapid collection of the sample.

Manipulation of the apparatus in obtaining the alveolar sample. The person conducting the determination watches the water manometer continuously. A, B, and $\mathrm{C}$ are filled with mercury, up to $\mathrm{K}$, before the determination is begun. With $F$ midway between 1 and $2, M_{2}$ is opened and the dead-space is washed out by turning $F$ to 1 during inspiration and to 2 during expiration. A convenient amount is 5 to $8 \mathrm{cc}$. per breath. Three such portions of air are withdrawn during 3 successive inspirations into $\mathrm{C}$ and discarded by ejection through the upper stopcock to room air. The sampling is immediately started by closing $\mathrm{M}_{2}$ and opening $M_{1}$. When $A$ is full, $M_{1}$ and $D$ are closed. During the second determination, both $\mathrm{C}$ and $B$ are taken, $C$ for analysis of carbon dioxide as a check on the method of collection of alveolar air. When respiration is irregular, as it may be in anesthetized patients, great care must be used to avoid taking a portion of air immediately after a respiration that is appreciably more shallow than 


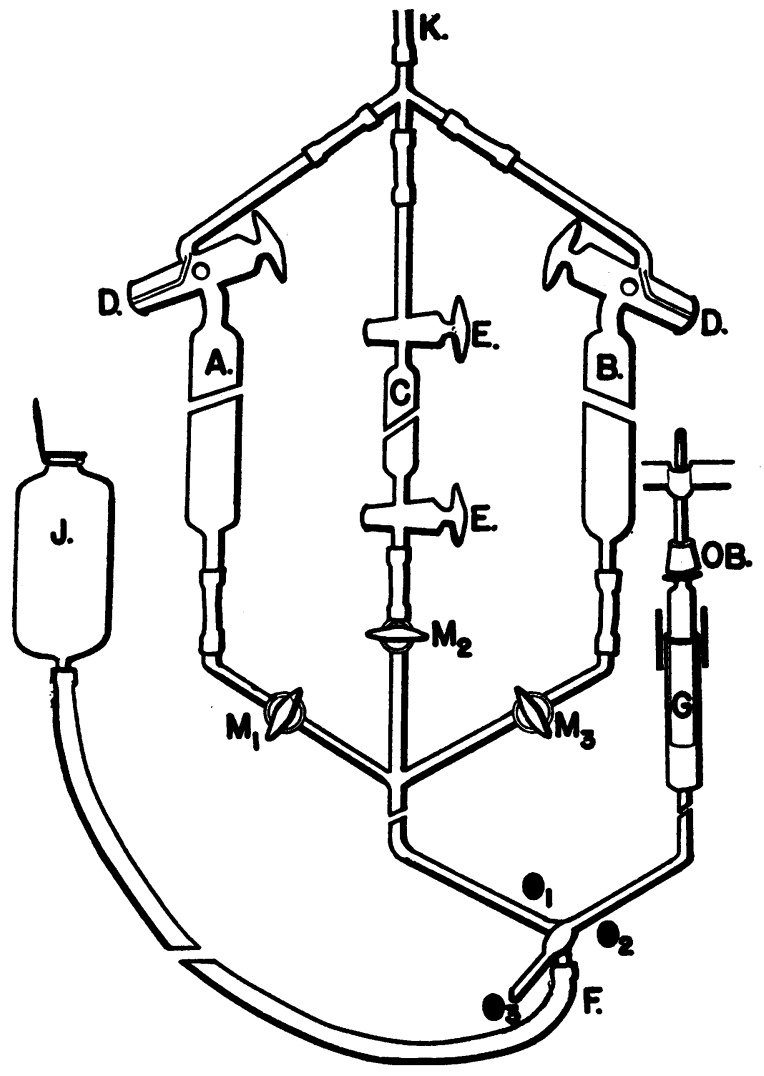

Fig. 1. Schema of Apparatus

A. and B. are calibrated tubes containing $200 \mathrm{cc}$. and fitted with stopcocks, D. D., which are inclined downwards about 30 degrees from the horizontal and are about $6 \mathrm{~cm}$. in length, with an outside diameter of 17 $\mathrm{mm}$. at the lower end. The bore of the plug is $4 \mathrm{~mm}$. for the connection between the manifold $K$. and the tube. The hole in the center of the plug which connects either $\mathrm{K}$. or the tube with outside air is $1 \mathrm{~mm} . M_{1}, M_{2}$, $M_{3}$ are $4 \mathrm{~mm}$. stopcocks. All tubing is of $7 \mathrm{~mm}$. glass, with glass to glass connections, excepting that between $\mathrm{F}$. and $\mathrm{J}$. which is nitrometer rubber tubing. C. is a sampling tube of $60 \mathrm{cc}$. capacity fitted with two three-way downward plug stopcocks, E. E., of $4 \mathrm{~mm}$. bore. F. is a Y-stopcock, $4 \mathrm{~mm}$. bore, with a metal strip attached to the handle of the plug; in Position 1, the mercury is allowed to flow from any of the tubes A., B., or C. into the Syringe G.; in Position 2, the mercury flows from G. into $\mathrm{J} . ;$ the dots at 1 and 2 indicate stops against which the metal strip on the stopcock plug handle strikes, thereby permitting the operator to control the flow of mercury without looking at the stopcock. Position 3 connects $\mathrm{J}$. and the tubes above during the transfer of samples. G. is a syringe barrel with an outlet of $7 \mathrm{~mm}$.; it is calibrated in $2 \mathrm{cc}$. amounts up to $20 \mathrm{cc}$. A collar of rubber tubing around the top permits a small amount of mercury to be added for a seal. OB. is an adjustable rubber obstacle against which the plunger of the syringe strikes when mercury is admitted from the tubes above. A water the others, or after the subject swallows, since one obtains thereby dead-space air which is high in ethyl iodide content. If the analysis for carbon dioxide in the alveolar sample is omitted, tube C may be used as a reservoir for dead-space air, a step which shortens the procedure by half a minute.

With this apparatus it is not necessary to counterweight the spirometer which may be balanced so that the subject is entirely unaware of effort during inspiration.

An important source of error in collecting the alveolar sample needs emphasis. Condensation of water above the rubber valve in the mouthpiece may seal the opening of the alveolar line at any point in the collection of the sample. A mercury manometer in the alveolar line immediately indicates occlusion of the orifice in the mouthpiece and permits adjustment of the valve at once.

\section{B. Procedure}

Starr and Gamble's procedure is followed in respect to the preliminary arrangements $(1,2)$. When the patient begins to breathe from the mouthpiece, the stopwatch is started and the spirometer reading is noted at the end of an expiration. Spirometer readings are recorded at minute intervals. Pulse rate and respirations are counted for 30 seconds alternately every other minute. In the interval between 0 and 3 minutes, the rebreathing bag is charged with room air (Section $I, C)$. Between 3 and 6 minutes the following operations are performed: The $I$ (inspired air) sample is taken and transferred (see Section II). The collection of the sample for determination of metabolic rate is adjusted so that the expired air from the center of the mixing bottle is drawn uniformly over the 3-minute period into the sampling tube. The dead-space in the alveolar line is washed out and the A (alveolar air) sample is collected, but not transferred. The E (expired air) sample is taken but not tranisferred. As quickly as possible thereafter, the valve in the mouthpiece is turned to the rebreathing bag at the end of an expiration. Time, spirometer reading,

manometer connected directly to the inspired air line between the mouthpiece and the rubber valve is not shown. There is no Bohr meter or Mueller valve in the circuit. 
and temperature are noted. After the rebreathing is completed, the patient is disconnected from the apparatus or the second determination is begun without pause. Regardless of which is done, the $\mathrm{R}$ (rebreathed air) sample is quickly taken and the $E$ and $R$ samples are transferred. If the patient has started to breathe for the second determination these operations and any other maneuvres necessary to begin the subsequent test can be performed in the preliminary 3-minute period, which also allows time for complete recovery of the respiration and circulation from any changes caused by the short rebreathing period. When the second estimation has been completed, both A samples are adjusted to the calibration mark at atmospheric pressure and transferred.

A sample for determination of metabolic rate is collected during each test, the second being used for the analysis, the first only if the second fails to check satisfactorily.

A mercury manometer was placed in the alveolar line to detect false alveolar samples. The determination of carbon dioxide was omitted. To determine the time required to wash out the deadspace in the expired line, room air was drawn through the apparatus for an hour, and a person of average size, under basal conditions, with a respiratory minute volume of 4 liters per minute, began to breathe through the mouthpiece. The per cent of oxygen in samples taken quickly from the center of the mixing bottle every minute showed no significant change after 3 minutes. That interval was chosen for the routine preliminary period.

The spirometer readings at the beginning of the sampling and at the start of the $R$ period are utilized for the calculation of the respiratory minute volume. If the readings at minute intervals indicate marked fluctuations, the determination is discarded.

\section{The rebreathing period}

Starr and Gamble (1, Figure 2) investigated the concentration of ethyl iodide in the rebreathing period after the subject had inspired from the spirometer mixture for 15 minutes. They found a slow decline, the 30 -second value being about 2 per cent less than the extrapolated 15 -second value. When the period of breathing ethyl iodide is short- ened to 6 minutes, it is possible that the tension of ethyl iodide in the venous blood will fall more rapidly during the $R$ period than it does when the tissues are more nearly saturated with ethyl iodide. To obtain data on this point, the following apparatus was used. A manifold with capillary jets was inserted between the mouthpiece and $R$ bag. Mercury sampling tubes calibrated at a mark on the stem were evacuated and attached to the manifold. Samples of air from the lung-bag system were taken rapidly at suitable intervals in the $\mathrm{R}$ period, and were adjusted to the calibration mark (at atmospheric pressure) before transfer to titration bottles. Results of 28 experiments on 2 normal subjects under basal and non-basal conditions may be stated briefly. When the bag contains a liter of air at the expected concentration of ethyl iodide and when the subject does not alter his respiration (rate 8 to 16 per minute) in any way in the $R$ period, the concentration of ethyl iodide rises sharply to a peak, falls somewhat less rapidly to 15 or 20 seconds, then declines at a uniform rate to 30 or 35 seconds, and decreases more rapidly thereafter. Under these circumstances, mixing probably does not occur before 20 seconds and samples up to that time do not indicate the vapor tension of ethyl iodide in the venous blood. Between 20 and 35 seconds the slope of the curve varies considerably; sometimes there is almost no change, sometimes there is a fall at 30 seconds to a value as much as 10 per cent below the 20 -second value. From these observations, it is concluded that the $R$ period in Starr and Gamble's procedure must be modified to eliminate the possible variations when a 6-minute breathing period is employed instead of the 15. A more accurate $R$ value can be obtained after a 6-minute period if the subject increases the rate and depth of respiration and terminates the rebreathing within 15 or 18 seconds, that is, before recirculation has occurred to an appreciable degree. It was found convenient to have the subject empty the bag completely 5 or 6 times in response to spoken directions. If room air is placed in the bag instead of a prepared ethyl iodide mixture, the time required for mixing is decreased.

If the subject has been inhaling from the spirometer for twelve minutes, the ethyl iodide concentration in the $R$ bag behaves in the same manner as described by Starr and Gamble (1, 
Figure 2). When the subject is not disconnected from the spirometer between determinations, the above modification is not necessary in the second and third $R$ periods, which may be conducted as in Starr and Gamble's procedure. On numerous occasions observations in triplicate revealed no greater difference in the cardiac output between the first and third estimations than is expected from the ordinary variations of the method (see Section III).

\section{MODIFICATION OF COOL'S METHOD FOR THE DETERMINATION OF ETHYL IODIDE}

The shaking and washing procedures in Cool's method for the determination of ethyl iodide (4) are eliminated as follows. The sample is collected by water displacement in a stopcock tube calibrated at $400 \mathrm{cc}$. (Figure 2). The source of air to be sampled is attached to arm $A$; the deadspace is drawn out through $B$, and the sample is admitted to the tube through arm $C$.

The flat-bottomed titration bottle is illustrated

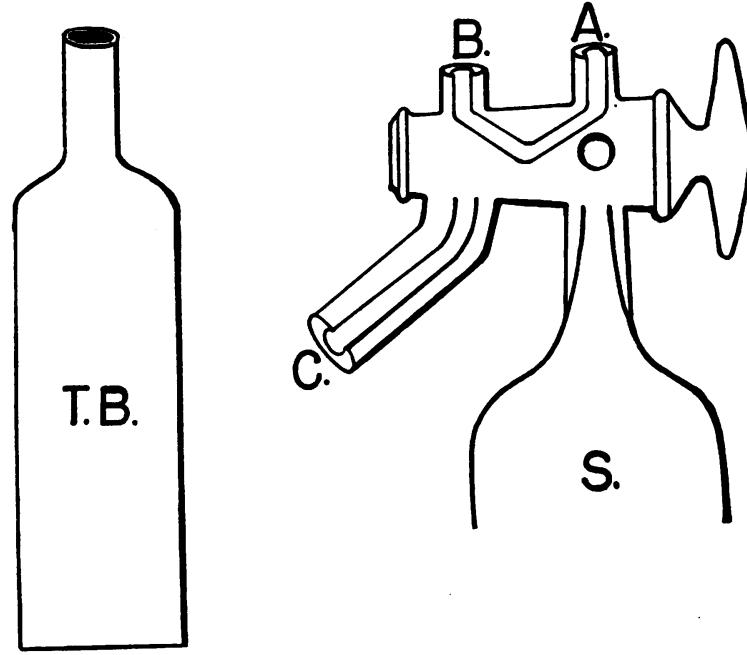

Fig. 2. Stopcock Tube and Titration Bottle

$\mathrm{S}$. is a sampling tube calibrated at $400 \mathrm{cc}$. The stopcock is constructed to connect the tube, S., and the arm marked A.; the tube and arm C.; and arms A. and B. Arm C. is $17 \mathrm{~mm}$. in outside diameter, and has a bore of $2 \mathrm{~mm}$. The stopcock bore between $A$. and $S$. is 4 $\mathrm{mm}$., between A. and B. (or C. and S.), $2 \mathrm{~mm}$.

T.B. is a flat-bottomed titration bottle which holds approximately $500 \mathrm{cc}$. The orifice at the top is 17 to $18 \mathrm{~mm}$. in diameter, a size which conveniently permits introduction of reagents required by the titration procedure. in Figure 2. The bottle is prepared by adding $10 \mathrm{cc}$. bromine water and evacuating with a water pump until the bromine water just begins to boil. (Twice the quantity of bromine water recommended by Cool is used since some bromine vapor is removed during evacuation.) A 4-inch section of rubber tubing ( $3 \mathrm{~mm}$. wall, $12 \mathrm{~mm}$. inside diameter) and a large hemostat or screw clamp seal the bottle. ${ }^{3}$

To transfer the sample from the stopcock tube to the titration bottle, the latter is fitted to arm $\mathrm{C}$, the hemostat is removed, and the bottle is pushed up into contact with the arm of the stopcock, which is then turned to connect the tube and titration bottle. Flow of water is controlled by a pinch clamp on the rubber tubing from the reservoir. Care must be taken to prevent any water from entering the titration bottle, since varying amounts of ethyl iodide are introduced, depending upon the number of times samples have been admitted to the tube. The rubber tubing is again securely clamped before the titration bottle is detached from $C$. The remaining procedures are carried out in another room free of ethyl iodide vapor (or after the windows have been opened) in order to prevent traces of ethyl iodide from being drawn into the bottles when the tubing is removed for titration (the pressure inside the bottles will be somewhat less than atmospheric if leaks have been avoided). After rotation for a minute or two, the walls of the bottle are washed down with $20 \mathrm{cc}$. distilled water; the remaining steps are carried out directly in the titration bottle following the directions given by Cool.

The principal source of error in this method is the possibility of loss of ethyl iodide into the displacing fluid. Starr and Gamble recommended (5, p. 526) 0.5 per cent nitric acid for a displac-

8 If the titration bottles are evacuated before the patient is allowed to breathe from the spirometer, it is advisable to seal the bottles with the blunt end of a test tube which is pushed part way into the rubber tubing before the hemostat is removed, and then into direct contact with the titration bottle. The 8 bottles necessary for duplicate determinations of output can be prepared easily in another room during the preliminary rest period while the patient is becoming basal. If test tube plugs are not used, an occasional leaky screw clamp or hemostat may allow sufficient air to enter the titration bottle during the few minutes of the test to prevent the accommodation of the entire $400 \mathrm{cc}$. sample. 
ing fluid. This was tried and found satisfactory at first. After a considerable number of determinations had been run using the same acid, consecutive samples from the spirometer began to show wide fluctuations in ethyl iodide content. Satisfactory results were obtained by replacing the nitric acid with distilled water. When freshly distilled water is the displacing fluid, analysis of air samples from the spirometer (containing ethyl iodide in the concentrations used in cardiac output determinations) regularly shows an average deviation of 0.9 to 1.5 per cent.

Another inaccuracy may be introduced if the samples are held in the collecting tube for varying lengths of time before transfer to a titration bottle. When a determination of cardiac output is run in duplicate, all the samples collected over water are transferred within a minute, and each is exposed for the same time as the corresponding sample in the other determination. The alveolar samples, being over mercury, are not transferred until a pair of tests is completed. On three occasions a sample was left in the collecting tube for 5 minutes before it was transferred. The values obtained did not differ from a previous series of samples by amounts in excess of the expected variation.

Volume of samples. The samples collected over water (I, E, R) are $100 \mathrm{cc}$. smaller than those in Starr and Gamble's procedure. The decrease in accuracy was accepted in the interest of convenience and rapidity. The alveolar samples, over mercury, were reduced to $200 \mathrm{cc}$. so that the amount of air taken with each respiration could be decreased. A greater margin of safety in turning the $Y$ stopcock is attained when only 5 to 10 cc. of air are collected per respiration instead of 10 to $20 \mathrm{cc}$. The samples over mercury can be adjusted to a known volume more accurately than those over water and show less variation.

\section{CARDIAC OUTPUT STUDIES ON NORMAL SUBJECTS AND HOSPITAL PATIENTS}

Starr and Gamble's procedure $(1,2)$ in preparing subjects for the determinations was followed. On several occasions the subjects slept in the laboratory bed and were studied soon after awakening. Hospital patients in most instances were familiarized with the apparatus by a dummy test conducted before the preliminary rest period, or under non-basal conditions on a different day.

Tables I and II contain data on the cardiac out-

TABLE I

\section{Basal cardiac outputs on normal subjects}

$\begin{array}{cc}\begin{array}{c}\text { Subject } \\ \text { number }\end{array} & \text { Date (in 1937) and cardiac output (liters per minute) } \\ 1 & \text { Jan. 14, 4.1, 3.4; Feb. 18, 2.9, 2.7; Mar. 25, 2.7, } \\ & \text { 3.3; May 7, 2.9, 3.1; May 12, 4.1, 3.6; May 26, } \\ & \text { 3.7, 2.6 } \\ 2 & \text { Dec. 8, 36, 4.6, 4.2; Mar. 4, 4.3, 4.2; Mar. 19, } \\ 3 & \text { 4.2; June 17, 4.3, 3.9 } \\ 4 & \text { Mar. 1, 2.7, 3.0; May 4, 3.8, 3.4; May 7, 3.7, 3.1 } \\ 4 & \text { Feb. 12, 3.6, 3.6; Mar. 30, 3.7, 3.5 } \\ 5 & \text { Mar. 27, 3.8, 4.2, 4.6; Apr. 17, 4.1, 4.4, 4.6 } \\ 6 & \text { Apr. 27, 3.8, 4.1; May 21, 4.6, 4.8 } \\ 7 & \text { May 28, 4.1, 4.2 } \\ 8 & \text { May 13, 5.3, 4.9 } \\ 9 & \text { May 26, 3.4, 3.2 }\end{array}$

\section{TABLE II}

\section{Basal cardiac outputs on hospital patients}

\begin{tabular}{|c|c|}
\hline $\begin{array}{l}\text { Patient } \\
\text { number }\end{array}$ & Date. (in 1937) and cardiac output (liters per minute) \\
\hline $\begin{array}{l}1 \\
2 \\
3 \\
4 \\
5 \\
6 \\
7\end{array}$ & $\begin{array}{l}\text { Jan. 18, 4.9, 5.1 } \\
\text { Feb. 19, 4.0, 4.3 } \\
\text { Jan. 9, 4.8, 5.4; Jan. 11, 6.3, 3.2 } \\
\text { Jan. 19, 7.0, 5.2; Jan. 22, 3.4, 3.5 } \\
\text { Jan. 29, 2.8, 3.4; Jan. 28, 3.3, 3.6; Feb. 3, 2.8, } 2.7 \\
\text { Mar. 12, 3.1, 4.2 } \\
\text { Mar. 23, 2.4, 3.1; Apr. 8, 2.5, 2.9 } \\
\text { Apr. 10, 3.4, 3.6; Apr. 11, 2.6, 3.3; Apr. 13, 4.2, }\end{array}$ \\
\hline $\begin{array}{r}9 \\
10\end{array}$ & $\begin{array}{l}\text { Apr. } 7,5.6,6.0 \\
\text { Apr. } 29,3.3,3.1 ; \text { Apr. 30, 2.9, 2.7; May 6, 3.3, }\end{array}$ \\
\hline $\begin{array}{l}11 \\
12 \\
13\end{array}$ & $\begin{array}{l}\text { Mar. 24, 3.6, 3.9 } \\
\text { Apr. 29, 3.1, 3.0; May } 29,4.2,4.4 \\
\text { May 18, 3.2, 3.5; May 20, 3.4, 3.5; May } 24,3.4 \text {, }\end{array}$ \\
\hline $\begin{array}{l}14 \\
15\end{array}$ & $\begin{array}{l}\text { Apr. 24, 3.8, 3.8; Apr. 28, 3.4, 3.6 } \\
\text { May 14, 2.3, 2.5; May 15, 2.5, 2.2, 3.5; May 17, } \\
\quad 2.8,2.8\end{array}$ \\
\hline $\begin{array}{l}16 \\
17\end{array}$ & $\begin{array}{l}\text { June } 8,4.7,4.8 ; \text { June } 9,5.8,4.4 \\
\text { June } 25,3.7,5.4,2.5 ; \text { June } 26,3.9,4.0\end{array}$ \\
\hline
\end{tabular}

put of 58 duplicate determinations on 9 normal subjects and 17 hospital patients. For the surgical patients, pre- and postoperative data are included for the purpose of enlarging the number of duplicate determinations used in calculating the average deviation of duplicate estimations from their mean. No data on patients under ether anesthesia are included.

For normal subjects the per cent average deviation of duplicates from their mean is 5.1 (19 duplicate and 2 triplicate estimations). The per cent deviation of each day's average about the mean of the daily averages is 5.4 (for the 6 normal subjects studied on more than one occasion). 
For hospital patients the per cent average deviation of duplicates from their mean is 8.3 (34 duplicate and 2 triplicate determinations). On three occasions, widely divergent results were obtained, and it is believed that the discrepancies were due to excitement of the subjects, since the second of the pair was much lower in each case. When these three pairs are omitted, the per cent average deviation of duplicates from their mean becomes 6.7. The per cent deviation of daily averages about their mean is 6.6 for 9 hospital patients studied on more than one occasion (24 determinations in duplicate; postoperative data obtained within 60 hours of operation and etherization are not employed in the calculations).

These results show that the present method as applied in the cases of this study is less accurate than Starr and Gamble's procedure. For comparison, their figures are stated (2): average deviation of duplicates about mean, normal subjects, 3.45 per cent; average deviation of duplicates about mean, hospital patients, 6.45 per cent ; average deviation of daily averages about their mean, normal subjects, 6.3 per cent.

The differences may be attributable to the small number in this series, and the fact that most of the patients were awaiting a surgical operation, as well as the variations in procedure which are introduced.

\section{DISCUSSION}

The changes in Starr and Gamble's method described in this communication are mechanical. They are such that the method may be applied to a wider variety of conditions. For example, five consecutive estimations of cardiac output can be made in 30 minutes, each one representing the circulation during a 3-minute period. The labor and time required for the analysis of samples by the chemical method are reduced.

The method involves more effort on the part of the persons conducting the test. The possibilities of error in collecting the alveolar sample are more numerous and require more careful attention. The rebreathing period in the first of a pair of estimations has been altered.

It should be pointed out that any of the modifications here suggested can be adopted alone. If it is held that the modified $R$ period is undesir- able, the objection can be entirely eliminated by waiting 15 minutes before taking samples for the first determination; additional determinations can still be made at 6-minute intervals as lonig as desired, the supply of glassware and the energy of the persons conducting the test being the only limiting conditions. If the mercury-syringe-stopcock for collection of alveolar air is not employed, the use of titration bottles saves time and labor in analysis nevertheless. And finally, if greater accuracy is required, larger samples and mercury as displacing fluid may be adopted at the expense of prolonging the estimations to 8 or 10 minutes each. Unfortunately, the katharometer method for the analysis of ethyl iodide (6) can be applied to repeated estimations at short intervals only if sets of collecting tubes equal in number to the consecutive determinations desired are provided.

As described, the method is particularly applicable to the study of cardiac output and oxygen consumption when these functions are undergoing changes which persist as long as 3 or 4 minutes.

\section{SUMMARY}

A modification of Starr and Gamble's ethyl iodide method for the study of cardiac output in man is described (Section I). A shorter, less laborious method for the collection and analysis of air samples is presented (Section II). Studies on normal subjects and hospital patients are cited (Section III).

The changes permit a duplicate determination of cardiac output to be made in 12 minutes and additional estimations every 6 minutes thereafter.

The method is somewhat less accurate than Starr and Gamble's procedure. Its advantages and limitations are discussed.

It is a pleasure to acknowledge the helpful suggestions given by Dr. Isaac Starr, Dr. C. J. Gamble, and Dr. M. D. Altschule, and the technical assistance of Miss Virginia Dewey and Miss A. B. Mangiaracine.

\section{BIBLIOGRAPHY}

1. Starr, I., Jr., and Gamble, C. J., An improved method for the determination of cardiac output in man by means of ethyl iodide. Am. J. Physiol., 1928, 87, 450. 
2. Starr, Isaac, Jr., Donal, J. S., Margolies, A., Shaw, R., Collins, L. H., and Gamble, C. J., Studies of the heart and circulation in disease; estimations of basal cardiac output, metabolism, heart size, and blood pressure in 235 subjects. J. Clin. Invest., 1934, 13, 561.

3. Starr, I., Gamble, C. J., Margolies, A., Donal, J. S., Jr., Joseph, N., and Eagle, E., A clinical study of the action of 10 commonly used drugs on cardiac output, work, and size; on respiration, on metabolic rate, and on the electrocardiogram. J. Clin. Invest., 1937, 16, 799.

4. Cool, R. D., Determination of small amounts of ethyl iodide. J. Biol. Chem., 1932, 97, 47.
5. Starr, I., Jr., and Gamble, C. J., A method for the determination of minute amounts of ethyl iodide in air, water, and blood by means of its reaction with silver nitrate; and experiments bearing on the determination of blood flow by means of ethyl iodide. J. Biol. Chem., 1927, 71, 509.

6. Donal, J. S., Jr., Gamble, C. J., and Shaw, R., The cardiac output in man. An adaptation of the katharometer for the rapid determination of ethyl - iodide in estimations of cardiac output by the ethyl iodide method. A study of the effect of posture upon cardiac output and other circulatory and respiratory measurements. Am. J. Physiol., 1934, 109, 666. 